\title{
Nonadiabatic quantum molecular dynamics with hopping. III. Photoinduced excitation and relaxation of organic molecules
}

\author{
M. Fischer, J. Handt, and R. Schmidt* \\ Institut für Theoretische Physik, Technische Universität Dresden, D-01062 Dresden, Germany
}

(Received 30 April 2014; published 29 July 2014)

\begin{abstract}
Photoinduced excitation and relaxation of organic molecules $\left(\mathrm{C}_{2} \mathrm{H}_{4}\right.$ and $\left.\mathrm{CH}_{2} \mathrm{NH}_{2}^{+}\right)$are investigated by means of nonadiabatic quantum molecular dynamics with hopping (NA-QMD-H), developed recently [Fischer, Handt, and Schmidt, paper I of this series, Phys. Rev. A 90, 012525 (2014)]. This method is first applied to molecules assumed to be initially ad hoc excited to an electronic surface. Special attention is drawn to elaborate the role of electron-nuclear correlations, i.e., of quantum effects in the nuclear dynamics. It is found that they are essential for a realistic description of the long-time behavior of the electronic relaxation process, but only of minor importance to portray the short-time scenario of the nuclear dynamics. Migration of a hydrogen atom, however, is identified as a quantum effect in the nuclear motion. Results obtained with explicit inclusion of an fs-laser field are presented as well. It is shown that the laser-induced excitation process generally leads to qualitatively different gross features of the relaxation dynamics, as compared to the field-free case. Nevertheless, the nuclear wave packet contains all subtleties of the cis-trans isomerization mechanism as observed without a laser field.
\end{abstract}

DOI: 10.1103/PhysRevA.90.012527

PACS number(s): 31.15.xv, 33.80.-b, 31.15.ee, 31.70.Hq

\section{INTRODUCTION}

The study of nonadiabatic processes in organic molecules has a long tradition [1-9]. The dynamics of ultrafast photochemical reactions is of genuine nonadiabatic character, like photochromism [10], where a structural rearrangement of the molecules changes its color, or photoisomerization, which is the fundamental mechanism for vision $[11,12]$. In addition, the control of isomerization processes of organic molecules gives rise to potential applications in material science or nanotechnology, e.g., for the manipulation of liquid crystals [13], holographic data storage [14], or molecular optomechanical engines [15].

Modern femtosecond (fs) laser technology and timeresolved spectroscopy make it possible to study the ultrafast internal conversions in real time. Numerous experiments have been performed for both small systems (e.g., acetylene [16]) studied by intense fs pulses from $\mathrm{x}$-ray-free electron lasers as well as larger systems (e.g., stilbene [17], azobenzene [14,18], or pyridine [19]) investigated with optical lasers.

Laser-induced isomerization reactions proceed generally in two steps: the initial electronic excitation and the subsequent nuclear and electronic relaxation. Thereby, the electronically excited system decays usually radiationless into its ground state via conical intersections and/or avoided crossings [20-22].

In most of the theoretical approaches the first step is not considered explicitly and the molecule is assumed to be initially, ad hoc, in an electronically excited state. One apparent scheme is to propagate the nuclear wave packet on coupled potential energy surfaces calculated in advance by quantum chemistry methods [5,23-25]. Evidently, in such approaches the number of considered degrees of freedom (DOFs) is limited. Alternatively, advanced "on the fly" $a b$ initio molecular dynamics (MD) methods have been

*Ruediger.Schmidt@tu-dresden.de applied, taking into account all nuclear DOFs. This includes the "semiclassical multiple spawning technique"[26-30], various classical MD approaches combined with time-dependent density functional theory (TD-DFT) for the electrons and Tully's trajectory surface hopping procedure for the nuclei [31-36] as well as mean-field MD [37-40].

There have been, however, also a few attempts to include the laser field explicitly in MD calculations and, thus, to treat excitation and isomerization on the same footing [4145]. Moreover, it has been shown that one can control the relaxation process by tuning the laser parameters [41,42]. So, besides isomerization, distinct fragmentation channels (fission, multifragmentation) can be observed by exciting selectively different bonds within the molecule, mainly by changing the laser frequency [41,42].

The investigations in Refs. [41,42] have been performed with the help of the nonadiabatic quantum molecular dynamics (NA-QMD) method developed [46] and permanently extended $[47,48]$ by us. In this theory, classical nuclear motion is coupled self-consistently to the electronic system using optionally TDDFT or time-dependent Hartree-Fock theory (TD-HF) in local basis expansion (as distinguished from grid-based methods $[49,50])$. The basis expansion technique allows us to treat systems as large as fullerenes in laser fields on an ab initio level. So the excitation of the giant vibrational breathing mode in $\mathrm{C}_{60}$ exposed to laser fields has been predicted by this method and was experimentally observed [51]. Recently large orientation effects in the energy absorption and subsequent relaxation channels of $\mathrm{C}_{60}$ in laser fields have been predicted as well [52]. For an overview of the broad range of NA-QMD applications see www.dymol.org.

Despite the universality of the NA-QMD method, it still contains physically a basic approximation: The nuclei are treated purely classically in the mean-field or Ehrenfest approximation. To overcome this limitation, we have recently basically extended the NA-QMD by including electron nuclear correlations with the help of Tully's surface hopping (NAQMD-H). The basic formalism of the NA-QMD-H approach 
has been given in Ref. [53] (hereafter cited as Paper I). A first application of the theory to atomic collisions has been presented in Ref. [54] (hereafter cited as Paper II), where the relevance of quantum effects in the nuclear dynamics in high energetic collisions between complex particles has been outlined.

In this work we apply the universal NA-QMD-H theory to the excitation and relaxation dynamics of organic molecules in laser fields with the following objectives:

(i) To verify the reliability of the new method, by comparing the results with existing quantum chemical calculations $[34,55]$

(ii) To reveal the general relevance of quantum effects in the nuclear dynamics on the electronic and nuclear relaxation processes in ad hoc excited molecules

(iii) To demonstrate the role of a possible laser excitation on the subsequent electronic and nuclear relaxation processes, realized under actual conditions in fs experiments.

The investigations are performed using two benchmark systems of photochemistry: the ethylene molecule $\mathrm{C}_{2} \mathrm{H}_{4}$, as a prototype of cis-trans isomerization [2], and the methaniminium cation $\mathrm{CH}_{2} \mathrm{NH}_{2}^{+}$, as the minimal retinal model [55].

The paper is organized as follows: In Sec. II basics and details of the calculations are outlined. In Sec. III the relaxation of the ad hoc excited methaniminium molecule is studied and compared with existing calculations. In Sec. IV the role of electron-nuclear correlations is elaborated, using the ad hoc excited ethylene molecule. In Sec. V the laser field is explicitly taken into account, and, thus, excitation and relaxation are treated on the same footings, starting with ethylene in its ground state. A brief summary and outlook are given in Sec. VI.

\section{BASICS}

First, the starting point of any time-dependent $a b$ initio study of finite atomic many-body systems is to describe their ground state structure reasonably. In nonadiabatic investigations, in addition, the electronically excited surfaces should be characterized realistically by the many-body approach. Here it is not our aim to compete with advanced quantum chemical configuration interaction (CI) methods, to describe subtleties of the nuclear structure and of the excited surfaces of the molecules, but, rather, to elaborate the essential physical aspects of the dynamics. Thus, in this work all investigations are performed using the spin-unrestricted Hartree-Fock theory (UHF), as also applied in our previous NA-QMD studies of organic molecules [41]. In the light of density functional theory (DFT), the UHF approach can be understood as a method with a nonlocal exchange (correlation) functional depending on the electronic density matrix instead of on the electronic density only [41]. From our experience we know that the UHF approach describes more realistically and more precisely the properties of the organic molecules under consideration than, for example, the local spin density approximation of DFT.

In Appendixes A and B, the geometry, the calculated ground state properties, and the relevant excited surfaces of both molecules, $\mathrm{CH}_{2} \mathrm{NH}_{2}^{+}$and $\mathrm{C}_{2} \mathrm{H}_{4}$, are presented. The results are compared with various CI calculations and available experimental data. Obviously the nuclear structural properties and electronically excited state energies reach quantum chemical accuracy. The calculated surfaces reflect all essential features to realistically describe the nonadiabatic dynamics that we are interested in in this work.

Second, in all time-dependent studies, the electronic and nuclear initial conditions must be unambiguously settled. Electronically the systems are assumed to be initially in the desired state, i.e., ground $\left(S_{0}\right)$, first excited $\left(S_{1}\right)$, or second excited state $\left(S_{2}\right)$. The classical initial positions and momenta of the nuclei have been sampled microcanonically according to the vibrational ground state (stretching and twisting modes) on the $S_{0}$ surface within the classical normal mode approximation. The results are then averaged over 50 trajectories with different initial conditions in the (deterministic) NA-QMD calculations, to take approximately into account the quantum nature of the initial nuclear state. In all (stochastic) NAQMD-H calculations, an additional 10 trajectories per classical initial condition, i.e., altogether $50 \times 10=500$ trajectories, are considered, to mimic the whole time-dependent nuclear wave packet. If the laser field is explicitly included, the $\mathrm{C}=\mathrm{C}$ bond of the molecule is assumed to be aligned along the laser polarization axis.

Finally, to characterize and to quantify the electronic and nuclear relaxation mechanisms, we will consider mainly four quantities as a function of time. This concerns the electrons, the mean electronic excitation energy $E_{\mathrm{ex}}^{\mathrm{el}}$, and the populations of the electronic eigenstates, i.e., the projections of the timedependent UHF-Slater determinant on the actual adiabatic electronic eigenstates $S_{0}, S_{1}$, and $S_{2}$. To characterize the nuclear dynamics, swarms of trajectories of the stretching bond length, $R_{\mathrm{CC}}$ or $R_{\mathrm{CN}}$, and the torsion angle $\theta$ are considered and presented as density plots for a significant visualization of the time-dependent wave packets.

\section{COMPARISON WITH EXISTING CALCULATIONS}

As mentioned, the methaniminium cation $\left(\mathrm{CH}_{2} \mathrm{NH}_{2}^{+}\right)$represents a simple model for retinal [55], which plays a basic role in the photoactivity of proteins and photoinduced cis-trans isomerization responsible for the primary process of vision. For this reason, it has attracted much attention in previous studies to shed light on retinal dynamics, on the one hand, and as a benchmark system to test new computational methods, on the other hand [33,34,37,39,55-57].

Despite its smallness, the methaniminium cation exhibits an interesting electronic structure leading to a complex excited state relaxation dynamics $[33,34,55,57]$. Excitation to the first excited state $S_{1}$ is followed by torsional motion around the CN double bond, leading to a fast decay to the ground state $S_{0}$, mainly due to the avoided crossing of the involved surfaces at $\theta=90^{\circ}$ (see Appendix A, Fig. 7). Excitation to the second excited state $S_{2}$, proceeds in a two-step process characterized by a fast decay of the $S_{2}$ to the $S_{1}$ state via $\mathrm{CN}$ bond stretching and repopulation of the ground state $S_{0}$ at later stages [34].

Here we use this molecule as a sensitive test case for a first NA-QMD-H application to the radiationless decay of organic molecules. As usually done in and to be able to compare with quantum chemical studies [33,34,55], the system is assumed to be initially ad hoc excited at the surface $S_{1}$ or $S_{2}$. The calculated NA-QMD-H results are shown in Figs. 1 and 2. 

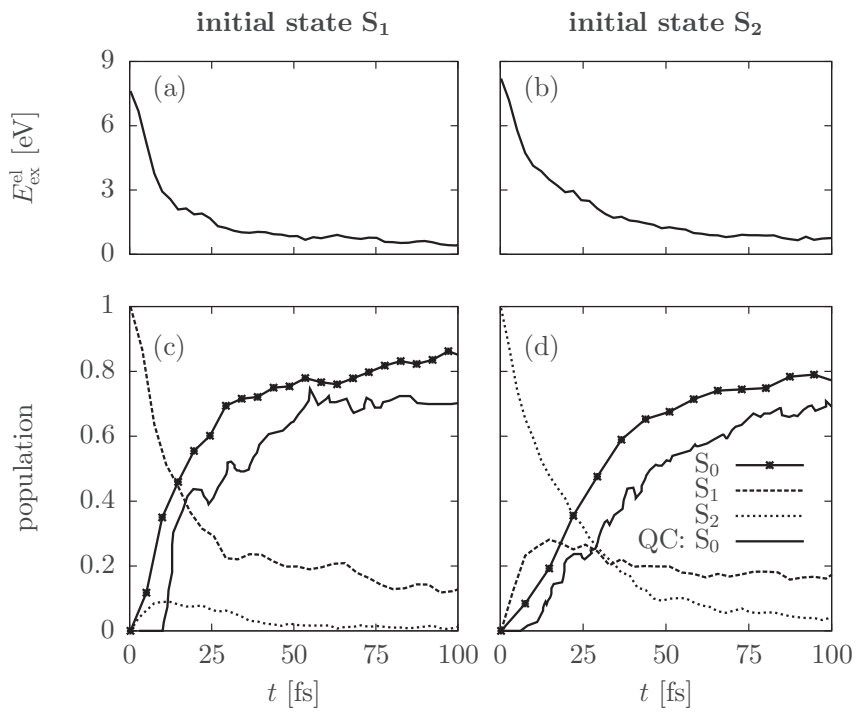

FIG. 1. NA-QMD-H electronic state relaxation dynamics of $\mathrm{CH}_{2} \mathrm{NH}_{2}^{+}$, initially excited to the $S_{1}$ state (left column) and the $S_{2}$ state (right column). Presented are the electronic excitation energies $E_{\mathrm{ex}}^{\mathrm{el}}(t)(\mathrm{a}, \mathrm{b})$ and the state populations for $S_{0}, S_{1}$, and $S_{2}(\mathrm{c}, \mathrm{d})$. The ground state populations, calculated with the quantum chemical code NEWTON-X (extracted from Ref. [34]), are also shown for comparison (QC: $S_{0}$ curves in c and d).

As can be seen in Figs. 1(a) and 1(b), the electronic excitation energies $E_{\mathrm{ex}}^{\mathrm{el}}$ decrease immediately in both cases, however, distinctly faster in the initial $S_{1}$ state, as it should be $[33,34,55]$. The general differences in and details of the electronic relaxation processes can be obtained from the time evolution of the populations, shown in Figs. 1(c) and 1(d). Starting from the $S_{1}$ state, the population decays directly to the ground state $S_{0}$. Excitation of the $S_{2}$ state is negligible [Fig. 1(c)]. Starting from the $S_{2}$ state, a fast decay mainly to the $S_{1}$ state during the first $20 \mathrm{fs}$ occurs, followed by a recovery of the ground state $S_{0}$, accompanied with a relatively long decay time for $S_{1}$.

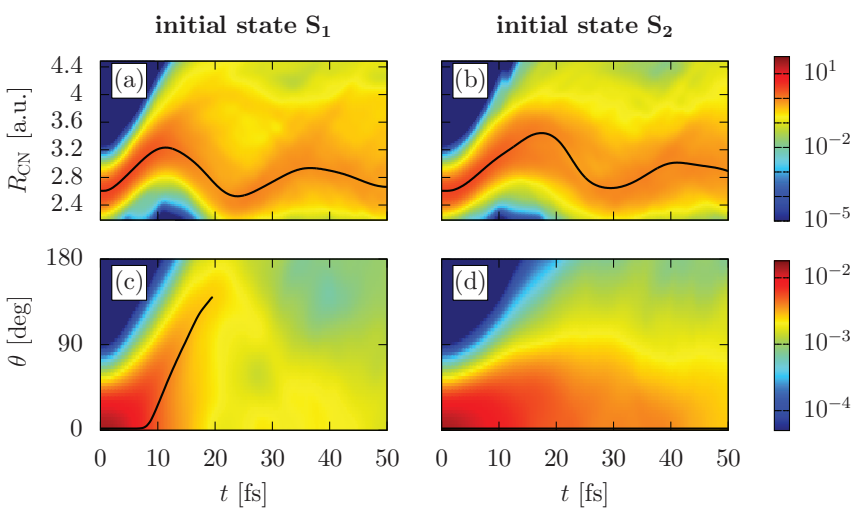

FIG. 2. (Color online) NA-QMD-H nuclear wave packet dynamics of $\mathrm{CH}_{2} \mathrm{NH}_{2}^{+}$, initially excited to the $S_{1}$ state (left column) and the $S_{2}$ state (right column). Shown are density plots of the classical trajectories along the $\mathrm{C}-\mathrm{N}$ bond length $R_{\mathrm{CN}}(\mathrm{a}, \mathrm{b})$ and the torsion angle $\theta$ (c, d) as a function of time $t$ (see Ref. [58]). The black curves indicate the maxima of the wave packets.
These observations are in very good agreement with previous findings [55]. In Figs. 1(c) and 1(d), we have added the $S_{0}$ populations, calculated with the quantum chemical program package NEWTON-X [34], for comparison. Regarding the differences in the methodologies of the present NA-QMD$\mathrm{H}$ and the NEWTON approach (hopping procedures, accuracy of the surfaces, etc.), the agreement is satisfactory and a good indication of the robustness and reliability of both methods.

In Fig. 2 the nuclear relaxation dynamics is characterized by drawing cuts of the 18-dimensional nuclear wave packet along the C-N bond stretching mode $R_{\mathrm{CN}}$ and the torsion angle $\theta$ as a function of time. Evidently from the beginning the $S_{1}$ surface relaxation process is dominated by the torsional mode. The maximum of the wave packet already reaches $\theta=180^{\circ}$ (i.e., the cis-trans transition) in about $20 \mathrm{fs}$ [see black line in Fig. 2(c)]. In the $S_{2}$ case, the torsional motion is active, but only a very small fraction of the wave packet undergoes cis-trans isomerization at $\theta=180^{\circ}$ [see green regions in Fig. 2(d)]. In both cases, however, the stretching mode $R_{\mathrm{CN}}$ plays a significant role with approximately harmonic behavior and slightly different frequencies [Figs. 2(a) and 2(b)]. Also this behavior is in excellent qualitative accord with previous conclusions $[33,34,55]$. A quantitative comparison of the wave packet dynamics, however, is naturally difficult to perform.

Altogether, the new NA-QMD-H approach reproduces the theoretical, hitherto known features of the electronic and nuclear relaxation mechanisms of the benchmark system methaniminium cation, assumed to be ad hoc excited to an electronic eigenstate. In fs-laser experiments, however, this idealized situation will never be reached, as will be demonstrated in Sec. V. In the next section, first, the general role of electron-nuclear correlations in the radiationless relaxation of organic molecules will be examined, using intentionally the ethylene molecule as an example.

\section{ROLE OF ELECTRON-NUCLEAR CORRELATIONS}

The role of electron-nuclear correlations (ENCs) in the theoretical description of nonadiabatic processes is of special interest for the very reason that many investigations (in particular, in atomic collisions and laser-molecule interaction) have been performed, so far, using the Ehrenfest approximation, i.e., neglecting all quantum effects in the nuclear dynamics. In Paper II, we have shown that ENCs are important to understand highly differential scattering observables, even in atom-atom collisions, where the de Broglie wavelength is much smaller than the interaction region and, thus naturally, quantum effects in the nuclear motion are expected to be negligible. In this section we will elaborate the general role of ENCs in photoexcited organic molecules using ethylene, as a further benchmark system.

The photoexcited dynamics of ethylene has been studied extensively using different methodologies [26-30,59-63], including various mean-field methods $[41,45]$. It is therefore an ideal candidate to investigate the relevance of ENC effects, in particular, to elaborate to what extent the conclusions drawn in Ehrenfest studies have to be possibly modified if ENCs are taken into account. To do so, we will compare the results obtained within the Ehrenfest MD (NA-QMD) and the surface hopping MD (NA-QMD-H). 

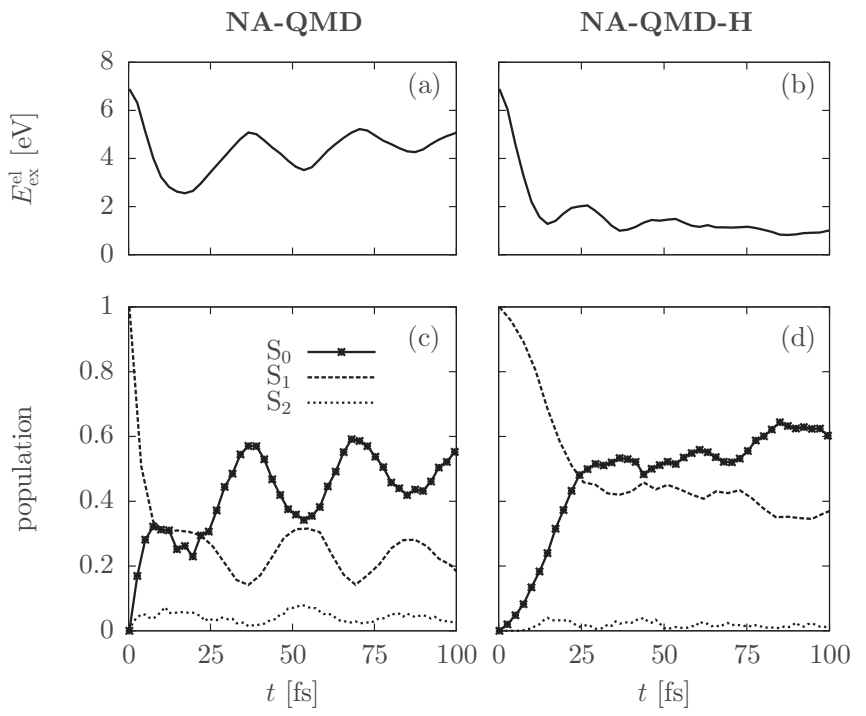

FIG. 3. Electronic state relaxation dynamics of $\mathrm{C}_{2} \mathrm{H}_{4}$, initially excited to the $S_{1}$ state, calculated with NA-QMD (left column) and NA-QMD-H (right column): Total electronic excitation energies $E_{\mathrm{ex}}^{\mathrm{el}}(t)(\mathrm{a}, \mathrm{b})$ and state populations for $S_{0}, S_{1}$, and $S_{2}(\mathrm{c}, \mathrm{d})$.

In a simplified picture, the photoinduced relaxation (isomerization) dynamics of ethylene can be discussed and understood in a three-state model, including the ground $\left(S_{0}\right)$, first $\left(S_{1}\right)$, and second $\left(S_{2}\right)$ excited states. As usual [26-30,59-63] the molecule is assumed to be ad hoc lifted to the $S_{1}$ state, with its nuclear geometry of the ground state $S_{0}$. The system evolves on the $S_{1}$ surface (mainly) via torsion and nonadiabatic transitions to the surfaces. De-excitation to the ground state proceeds favorably via a $S_{1}-S_{0}$ conical intersection at $\theta=90^{\circ}$ (see Appendix B, Fig. 8).

The results of the dynamical calculations, including many surfaces, are presented in Figs. 3 and 4. Initially the electronic excitation energy $E_{\mathrm{ex}}^{\mathrm{el}}$ decreases rapidly, due to electron-

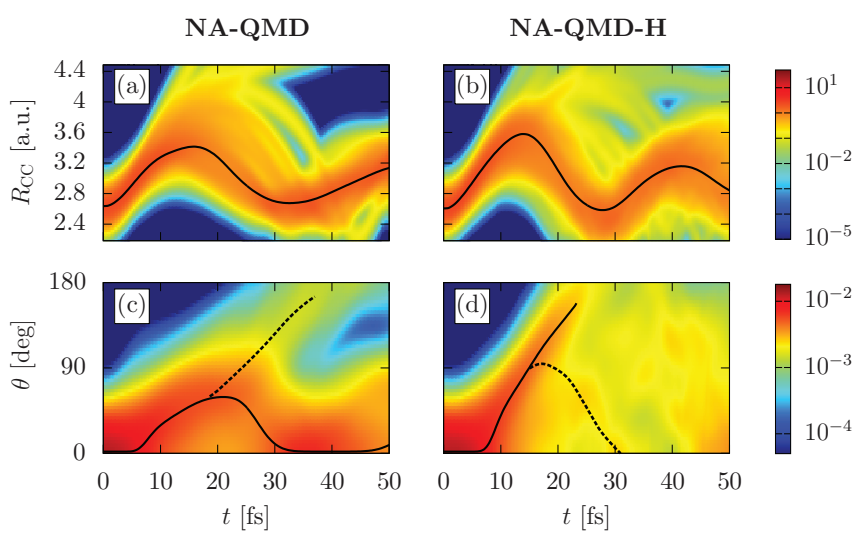

FIG. 4. (Color online) Nuclear dynamics of $\mathrm{C}_{2} \mathrm{H}_{4}$, initially excited to the $S_{1}$ state, calculated with NA-QMD (left column) and NA-QMD-H (right column). Shown are density plots of the classical trajectories along the $\mathrm{C}-\mathrm{C}$ bond length $R_{\mathrm{CC}}(\mathrm{a}, \mathrm{b})$ and the torsion angle $\theta$ (c, d) as function of time $t$ (see Ref. [58]). The full black lines are the total maxima of the densities. The dashed black lines in panels $\mathrm{c}$ and $\mathrm{d}$ are side maxima. vibrational coupling, in both approaches [Figs. 3(a) and 3(b)]. The long-time behavior of the electronic excitation energy, however, is basically different in both cases. In the correlated NA-QMD-H dynamics, this quantity practical continuously decreases [Fig. 3(b)]. In the mean-field NA-QMD case, $E_{\mathrm{ex}}^{\mathrm{el}}$ clearly increases after about $20 \mathrm{fs}$, and afterwards oscillates, but with a clear tendency of a further increase in the mean [Fig. 3(a)].

This puzzling increase of the electronic excitation energy during the long-time relaxation is a rather general feature of the nonadiabatic mean-field dynamics and already has been observed also in other systems, like metallic clusters or fullerenes $[64,65]$. From the present studies, it becomes apparent that this effect results from permanent transitions to higher excited states during the nuclear mean-field motion. The hopping mechanism prevents these unphysical transitions. This can be seen in the lower part of Fig. 3, where the time evolution of the populations is shown. Evidently in the NA-QMD-H calculations, the sum of the populations $S_{0}, S_{1}$, and $S_{2}$ remains almost one [Fig. 3(d)] and, thus, corresponds to the three-state picture of the dynamics given above. In the NA-QMD calculations, already after about $10 \mathrm{fs}$, it becomes clearly smaller than one [Fig. 3(c)] and, thus, higher surfaces are involved (not shown). Even if their population probability is small, they do have a large influence on the electronic energy and, thus, on the total energy balance (see discussion of the nuclear dynamics below).

From an exponential fit, $f(t)=\exp \left(-\frac{t}{\tau}\right)$, of the $S_{1}$ population in Fig. 3(d) one can estimate a decay time of this state of about $\tau \approx 160$ fs which lies in the region of decay times reported in other theoretical studies $(\tau \approx 89-180 \mathrm{fs}$; see Refs. [26,61,66,67]). We note that the long existing discrepancy between the theoretically calculated lifetimes and the experimentally measured one of $\tau \approx 50 \mathrm{fs}$ has been resolved recently $[29,30]$ and originates from the impact of the instantaneous ionization potential of the molecules in the pump-probe experiments on the measured lifetimes.

In Fig. 4 the corresponding nuclear dynamics is presented by considering density plots of the classical trajectories along the $\mathrm{C}=\mathrm{C}$ bond length $R_{\mathrm{CC}}$ and the torsion angle $\theta$. The molecular motion starts on the $S_{1}$ state with a geometry near the planar equilibrium ground state structure with bond length $R_{\mathrm{CC}} \approx 2.6$ a.u. and torsion angles around $\theta \approx 0$. During the first stage of the evolution (times up to about 20-25 fs), the system is strongly driven to the conical intersection at $\theta \approx 90^{\circ}$ by torsional motion on the $S_{1}$ surface [Figs. 4(c) and $4(\mathrm{~d})$ ], accompanied by a distinct $\mathrm{C}=\mathrm{C}$ bond stretching oscillation [Figs. 4(a) and 4(b)], owing to a minimum of the $S_{1}$ surface at larger $R_{\mathrm{CC}}$ (not shown here). This holds for both calculations with the tendency that the wave packet dynamics of the NA-QMD-H calculations is somewhat faster owing to the general larger vibrational kinetic energy (see discussion below). At the conical intersection, quenching to the ground state occurs via nonadiabatic transitions [see Figs. 4(c) and 4(d)] with subsequent nuclear relaxation, back either to the cis-isomer $(\theta=0)$ or to the trans-isomer $\left(\theta=180^{\circ}\right)$, again observed in both cases.

The cis-trans branching ratios, however, are basically different. Whereas most of the classical NA-QMD trajectories are driven back to the $c i s$-state, the opposite is the case for the 
NA-QMD-H wave packet, whose total maximum clearly moves to the trans-state [see solid black lines in Figs. 4(c) and 4(d)]. The origin of this effect is the qualitative different behavior of the electronic energies as discussed above [Figs. 3(a) and 3(b)]. The physically correct electronic relaxation (deexcitation) in the correlated NA-QMD-H description leads generally to a larger vibrational kinetic energy at sufficiently long times, as compared to the mean-field NA-QMD treatment. In the latter case, the unphysical continuous increase of the electronic excitation energy leads finally to a strong reduction of the nuclear mobility. This can be seen nicely in Figs. 4(a) and $4(\mathrm{c})$ at longer times. The bond stretching $R_{\mathrm{CC}}$ amplitude becomes permanently smaller as a function of time, and, the torsional motion is restricted to oscillations around $\theta=0$ [see deep blue areas of missing densities in upper right corners in Figs. 4(a) and 4(c)]. The nuclear NA-QMD-H wave packet, on the contrary, is smeared out over the whole range of $\theta$ and exhibits a distinctly larger bond stretching amplitude at times between 40 and 50 fs [Figs. 4(b) and 4(d)]. The already existing damping of the $R_{\mathrm{CC}}$ oscillation [black line in Fig. 4(b)] results from the coupling to the other nuclear DOF (not shown), rather than from electronic effects.

Finally we note that hydrogen-migration has been reported in the literature as a relevant dynamical pathway in ethylene as a precursor of $\mathrm{H}_{2}$ elimination [28,60,62,68]. From our analysis one can conclude that this process is a pure quantum phenomenon of the nuclear motion, because it has never been observed in NA-QMD simulations, whereas it belongs to typical trajectories in the long-time behavior of the NAQMD-H calculations (see Fig. 5).

To summarize this section, ENCs are of general importance to describe realistically the long-term behavior of nonadiabatic relaxation processes. In particular, they prevent the unphysical increase of the electronic excitation energy, which is an inherent failure of the Ehrenfest dynamics. In addition, migration of hydrogen atoms is identified as a typical quantum relaxation phenomenon. On the other side, ENCs are of minor relevance for the short-time dynamics of the nuclei. Nevertheless, they influence considerably the cis-trans isomerization branching ratio and probability for this reaction channel.

\section{ROLE OF THE INITIAL LASER EXCITATION}

So far the nonadiabatic relaxation dynamics of organic molecules have been considered, assuming the molecule initially to be ad hoc excited to a particular surface. This approach corresponds to the typical issues of traditional photochemistry $[3,4,9]$, where the initial excitation process is not of interest, a priori given by the absorption of a single photon, and, thus, is basically not influenceable.

In the actual physical research, however, it is just the excitation mechanism which is of most central interest, in order to control the relaxation dynamics of molecules or clusters $[69,70]$. For example, the existence of the giant breathing mode in $\mathrm{C}_{60}$ has been detected by optimizing the laser parameters in pump-probe experiments [51]. Very recently, it has been found that the outcome of fragmentation reactions in ethylene depends strongly on the laser parameters [71]. This phenomenon has been predicted for ethylene [41] (and diimide [42]) in previous NA-QMD calculations. The present, extended NA-QMD-H formalism also allows us to explicitly take into account an external laser field and, thus, to study excitation and relaxation on the same footing on this level of approximation (Paper I).

In this section we present an application of the NA-QMD$\mathrm{H}$ method including an external fs-laser field by considering the dynamics of ethylene. The aim is to reveal, first, general aspects of an initial laser excitation on the nuclear (hopping) relaxation, in particular, on the cis-trans isomerization process in our example. To this end, one needs a laser field in which as large as possible population of the $S_{1}$ surface occurs. This will allow us to compare the results for the relaxation with that of the field-free calculations presented in the previous section.

The system starts initially from the ground state $S_{0}$. At $t=0$ it is exposed to a $\sin ^{2}$-shaped laser field

$$
\mathbf{E}(t)= \begin{cases}\mathbf{E}_{0} \sin ^{2}\left(\frac{\pi t}{T}\right) \cos (\omega t) & \text { for } 0 \leqslant t \leqslant T \\ \mathbf{0} & \text { otherwise }\end{cases}
$$

with a total pulse length $T=10 \mathrm{fs}$, fundamental frequency $\omega=7.07 \mathrm{eV}$, and peak intensity of $I=\frac{\mathbf{E}_{0}^{2}}{2}=3 \times 10^{12} \frac{\mathrm{W}}{\mathrm{cm}^{2}}$. These laser parameters have been carefully chosen. The short pulse length is mandatory because the inclusion of a laser field in the theory is restricted to time scales where the nuclear motion remains practically frozen (see Paper I for details). The frequency lies in the vicinity of the $S_{0}-S_{1}$ excitation energy of $\Delta E=7.20 \mathrm{eV}$ (see Table II). It does not correspond exactly to this value, calculated for the nuclear ground state geometry, because the nuclear initial conditions include stretching and twisting displacements from this geometry (see Sec. II). The relatively low intensity, the only parameter which arbitrarily can be varied now, guarantees (at least to a large extent; see below) that the excitation of higher excited states is avoided. The results of the calculations are summarized in Fig. 6.

As can be seen, the electronic excitation energy now increases as long as the laser pulse is active [see maximum of $E_{\mathrm{ex}}^{\mathrm{el}}$ at $t=10 \mathrm{fs}$ in Fig. 6(a)]. During this period the ground state $S_{0}$ population decreases and mainly the first excited state $S_{1}$ (up to about $20 \%$ of the total population), and partly also the second excited state $S_{2}(5 \%)$ becomes populated. An, in principle, desired increase of the $S_{1}$ population by increasing the laser intensity is automatically connected with an unavoidable and undesired increase of the $S_{2}$ and higher excited states' populations. After the pulse, the system deexcites with a ground state revival time of about $100 \mathrm{fs}$ [Fig. 6(b)]. From this basically different behavior as compared to that of the field-free case [cf. Figs. 3(b) and 3(d)], one can hardly expect similarities in the nuclear relaxation.

At first glance, this seems indeed to be the case [see gross features in Figs. 6(c) and 6(d) and 4(b) and 4(d)]. The uppermost part of the nuclear wave packet stays on the electronic ground state surfaces $S_{0}$ around the equilibrium bond length of $R_{\mathrm{CC}}=2.6$ a.u. and at angles $\theta$ between $\theta=0$ and $\theta=45^{\circ}$ [dark red regions in Figs. 6(c) and 6(d)]. However, and somewhat surprisingly, a small but finite part of the wave packet shows all characteristics of the cis-trans isomerization process on the $S_{1}$ surface, as known from the previous field-free calculations [cf. Fig. 4(b) and 4(d) and yellow branches in Figs. 6(c) and 6(d)]. The stretching mode is excited with the same frequency and damped amplitude, as before [Figs. 4(b) 

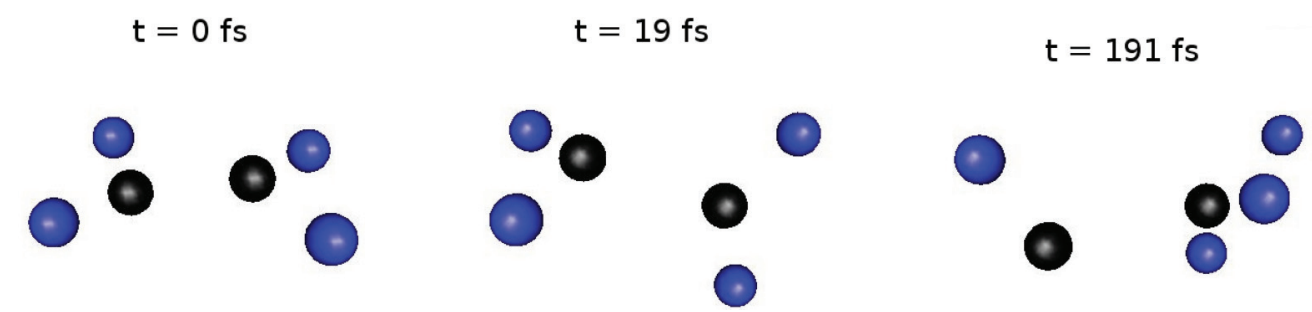

FIG. 5. (Color online) Typical trajectory for the excited state relaxation of ethylene calculated with NA-QMD-H: the system is initially in the first excited state $S_{1}$ with the near-planar geometry of the ground state (first snapshot, $t=0 \mathrm{fs}$ ) and evolves via torsional motion and $\mathrm{C}-\mathrm{C}$ bond stretching (second snapshot, $t=19 \mathrm{fs}$ ). At later times, hydrogen migration occurs to form ethylidene-like structures (third snapshot, $t=191 \mathrm{fs}$ ), which is a potential precursor for subsequent $\mathrm{H}_{2}$ elimination.

and Fig. 6(c)]. The angular parts of the wave packet reach the trans-angle $\theta=180^{\circ}$ at the same time scale of about $25 \mathrm{fs}$ [cf. Fig. 4(d) and yellow bunch in Fig. 6(d)]. Such subtleties of the laser-induced cis-trans dynamics can hardly be observed in a mean-field description of the nuclei.
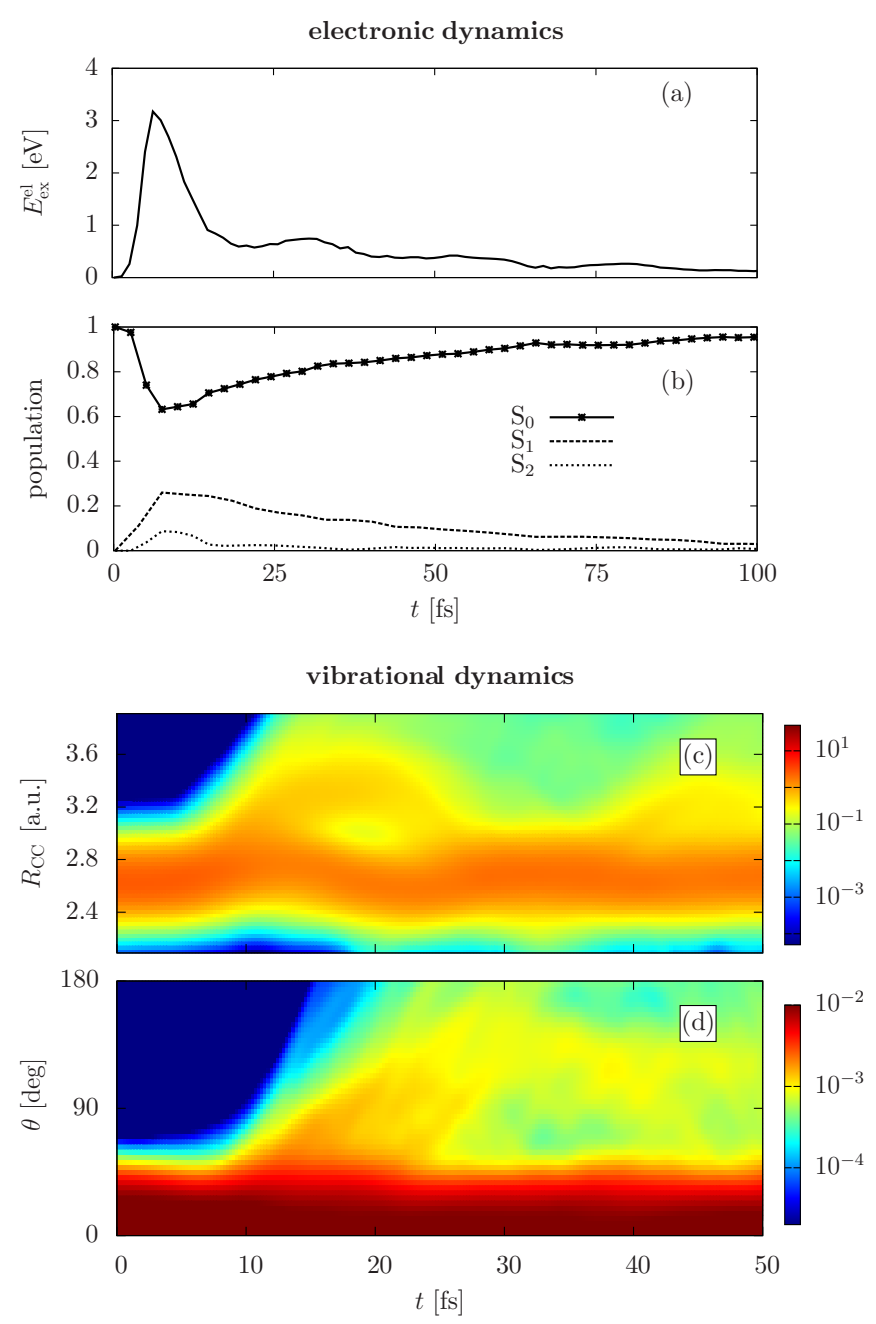

FIG. 6. (Color online) Laser-induced excitation and relaxation dynamics of $\mathrm{C}_{2} \mathrm{H}_{4}$. Shown are, as function of time $t$, the total electronic excitation energy $E_{\mathrm{ex}}^{\mathrm{el}}(t)$ (a), the state populations for $S_{0}$, $S_{1}$, and $S_{2}$ (b), the nuclear densities along the C-C bond length $R_{\mathrm{CC}}$ (c), and the torsion angle $\theta$ (d).
Nevertheless and concluding this part, one can state that in a short laser pulse basically several surfaces are involved from the very beginning. The nuclear relaxation proceeds unavoidably on different surfaces. To enhance the cis-trans yield of ethylene demands mandatory the optimization of the laser parameters for sufficient long pulses (i.e., up to pulse durations, which include the nuclear motion). To predict these parameters requires a basic extension of the present NA-QMD-H formalism, in which (maybe) the nuclei move on and hop between Floquet surfaces [72-74] or other light-induced potentials [75,76] instead of Born-Oppenheimer surfaces (Paper I). To what extent this conclusion remains valid for other, ultrafast reaction channels proceeding on higher excited states, like fission or multifragmentation [41,42,71], remains an interesting subject of future studies.

\section{SUMMARY}

We presented a systematic application of the recently developed NA-QMD-H method (Paper I) to the photoinduced dynamics in organic molecules.

To test the reliability of the method, we first applied it to the methaniminium molecule, $\mathrm{CH}_{2} \mathrm{NH}_{2}^{+}$, assumed to be $\mathrm{ad}$ hoc excited to the first and second electronic eigenstate. For this benchmark system, recorded data about the relaxation processes are very well known from numerous other $a b$ initio studies [33,34,55,57], and our results are in very good agreement with these findings.

Second, we elaborated the role of ENC effects in the relaxation dynamics by comparing the NA-QMD-H results with that of mean-field (i.e., NA-QMD) calculations for ethylene $\mathrm{C}_{2} \mathrm{H}_{4}$, assumed to be initially ad hoc excited to the $S_{1}$ surface. First, ENCs prevent the permanent increase of the electronic excitation energy, which is an inherent unphysical feature of the long-time Ehrenfest dynamics, leading to an ongoing cooling of the nuclear system. In consequence, hydrogen migration is forbidden in the Ehrenfest dynamics, which, on the other side, represents a typical reaction channel if ENCs are taken into account. The short-time behavior of the nuclear dynamics, however, in particular the cis-trans isomerization mechanism, is much less affected by correlation effects.

Finally, we studied the dynamics of ethylene in a fs-laser field, treating excitation and relaxation on the same footing, with a special emphasis on the investigation of the cistrans isomerization process. In this case several surfaces are involved. With carefully chosen laser parameters, a maximum 
$S_{1}$ population of about $20 \%$ could be achieved, and, thus, the upper most part of the nuclear density remains on the ground state surface $S_{0}$ after laser excitation. Nevertheless, the foothills of the wave packet contain all details of the cis-trans mechanism on the $S_{1}$ state as observed in the field-free case. To enhance the cis-trans yield requires optimization of the laser parameters for long-time scales, which include the nuclear motion.

\section{ACKNOWLEDGMENTS}

We gratefully acknowledge the allocation of computer resources from the ZIH of the Technische Universität Dresden and the financial support from the Deutsche Forschungsgemeinschaft through the Normalverfahren.

\section{APPENDIX A: STRUCTURE AND SURFACES OF THE $\mathrm{CH}_{2} \mathrm{NH}_{2}^{+}$MOLECULE}

The calculations are performed using a minimal basis set including the $1 s$ and $2 s$ atomic orbitals of hydrogen as well as the $2 s$ and $2 p$ atomic orbitals of carbon and nitrogen. The two innermost electrons of the latter are treated within the frozen core approximation. The atomic and the corresponding density basis sets are constructed from the 6-31G and DeMon Coulomb Fitting basis sets [77-79] using the $s$ - and $p$-type Gaussians only.

Table I shows results for the planar ground state equilibrium structure (with $\mathrm{C}_{2 \mathrm{v}}$ symmetry) and the corresponding vertical electronic excitation energy of the first excited state $S_{1}$. Thereby, an electronic excitation in the NA-QMD-H method corresponds to single-particle excitations according to the involved excited adiabatic Slater determinant (for more details see Paper I), i.e., in this work (spin-unrestricted) Hartree-Fock single-particle excitations.

TABLE I. Ground state structure of $\mathrm{CH}_{2} \mathrm{NH}_{2}^{+}$as well as the first optically allowed $\left(S_{0} \rightarrow S_{1}\right)$ excitation energy $\Delta E$. In addition, RHF-CIS results are shown calculated with GAMESS [80] using the 6-31G basis set [77-79]. In addition, accurate quantum chemical values (MR-CISD) are taken from Ref. [55]. For the definition of the geometry parameters see the sketch at the top.
$\mathrm{H}$

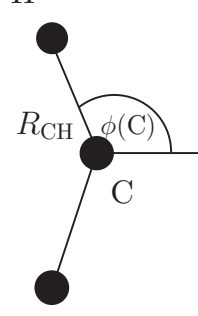

$\mathrm{H}$

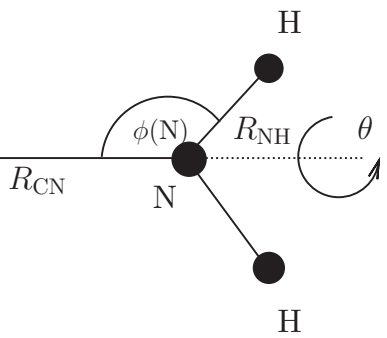

\begin{tabular}{lcccccc}
\hline \hline & $\begin{array}{c}R_{\mathrm{CN}} \\
(\AA)\end{array}$ & $\begin{array}{c}R_{\mathrm{CH}} \\
(\AA)\end{array}$ & $\begin{array}{c}R_{\mathrm{NH}} \\
(\AA)\end{array}$ & $\begin{array}{c}\phi(\mathrm{C}) \\
(\mathrm{deg})\end{array}$ & $\begin{array}{c}\phi(\mathrm{N}) \\
(\mathrm{deg})\end{array}$ & $\begin{array}{c}\Delta E \\
(\mathrm{eV})\end{array}$ \\
\hline UHF (this work) & 1.38 & 1.08 & 0.98 & 118.8 & 120.7 & 7.86 \\
RHF-CIS & 1.27 & 1.07 & 1.00 & 120.2 & 122.2 & 8.82 \\
MR-CISD $^{\mathrm{a}}$ & 1.27 & 1.08 & 1.01 & 119.4 & 121.5 & 8.47 \\
\hline \hline
\end{tabular}

${ }^{\mathrm{a}}$ See Ref. [55].

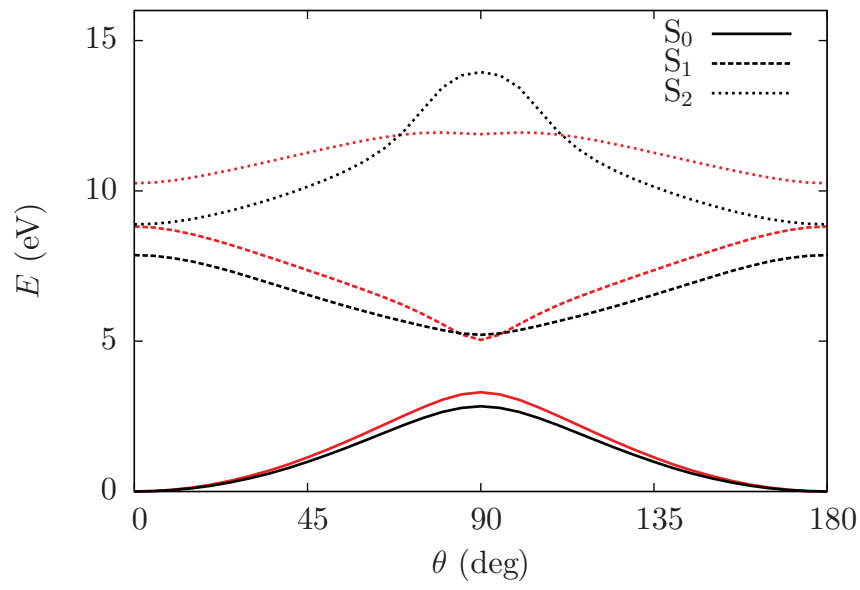

FIG. 7. (Color online) Potential energy surfaces of $\mathrm{CH}_{2} \mathrm{NH}_{2}^{+}$for the ground state $S_{0}$ and the lowest excited states $S_{1}$ and $S_{2}$ dependent on the torsion angle $\theta$ calculated using UHF (this work) (black curves) and RHF-CIS (GAMESS) (red curves). All remaining geometry parameters are kept frozen according to the ground state equilibrium values.

We compare the UHF values of this work with spinrestricted Hartree-Fock results combined with a configuration interaction single excitation approach to determine (besides the ground state geometry) the electronic excitation energy (RHF-CIS, 6-31G basis set) calculated with GAMESS [80], and additionally with highly accurate quantum chemical results, in particular with multireference configuration interaction with single- and double-excitation (MR-CISD) calculations taken from Ref. [55].

Table I shows that the $\mathrm{CH}$ and $\mathrm{NH}$ bond length $\left(R_{\mathrm{CH}}\right.$ and $\left.R_{\mathrm{NH}}\right)$ and the bond angles $[\phi(\mathrm{C})$ and $\phi(\mathrm{N})]$ are described quite well. In contrast, the UHF results for the $\mathrm{CN}$ bond length $R_{\mathrm{CN}}$ and the excitation energy $\Delta E$ of the first excited state $S_{1}$ deviate slightly from the exact ones. This deviation is, however, less than $10 \%$.

The potential energy curves of the ground and the first two excited states in dependence on the torsion angle $\theta$ are shown in Fig. 7 (black lines). For comparison, the potential energy curves calculated with the RHF-CIS approach are also plotted (red lines). The ground and first excited state surfaces $S_{0}$ and $S_{1}$ agree quite well qualitatively with the RHF-CIS results, exhibiting a maximum of the ground state and minimum of the first excited state at the twisted orthogonal structure $\left(\theta=90^{\circ}\right)$ with an energy gap of $2.5 \mathrm{eV}$.

In contrast, the second excited state surface $S_{2}$ agrees qualitatively only around the planar ground state equilibrium geometry $(\theta \approx 0)$ and deviates for larger torsion angles. This is expected to be a minor problem in the dynamical calculations, however, as the decay from the $S_{2}$ state proceeds very fast mainly via $\mathrm{CN}$ bond stretching (see also Ref. [34]), and, thus, larger torsion angles are unlikely to be accessed as the system evolves on this state.

\section{APPENDIX B: STRUCTURE AND SURFACES OF THE $\mathrm{C}_{2} \mathrm{H}_{4}$ MOLECULE}

In the calculations with $\mathrm{C}_{2} \mathrm{H}_{4}$, the same basis functions for the hydrogen and carbon atoms are used as for the methaniminium cation (see Appendix A). 
TABLE II. Ground state structure of $\mathrm{C}_{2} \mathrm{H}_{4}$ as well as the first optically allowed $\left(S_{0} \rightarrow S_{1}\right)$ excitation energy $\Delta E$. In addition, RHF-CIS results are shown calculated with GAMESS [80] using the 6-31G basis set [77-79]. The table contains also accurate quantum chemical results (MR-CISD) and experimental data. For the definition of the geometry parameters see the sketch at the top.

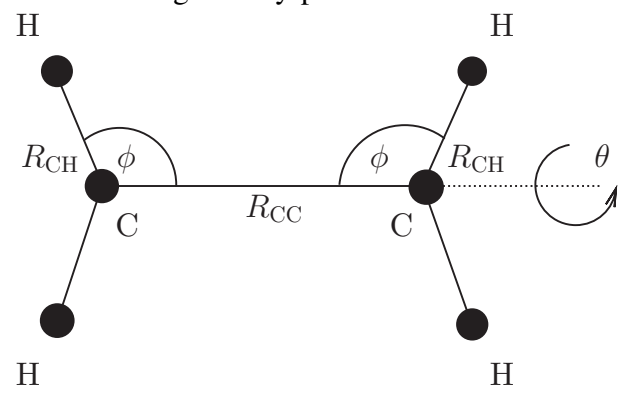

\begin{tabular}{lcccc}
\hline \hline & $R_{\mathrm{CC}}(\AA)$ & $R_{\mathrm{CH}}(\AA)$ & $\phi(\mathrm{deg})$ & $\Delta E(\mathrm{eV})$ \\
\hline UHF [this work] & 1.39 & 1.07 & 120.9 & 7.20 \\
RHF-CIS & 1.32 & 1.07 & 121.9 & 8.82 \\
MR-CISD $^{\mathrm{a}}$ & 1.34 & 1.08 & 121.5 & 7.8 \\
Experiment $^{\mathrm{b}}$ & 1.34 & 1.07 & 121.2 & 7.66 \\
\hline \hline
\end{tabular}

${ }^{\mathrm{a} S e e}$ Ref. [60].

${ }^{\mathrm{b}} \mathrm{See}$ references in Ref. [27].

The results for the optimized planar ground state equilibrium structure (with $\mathrm{D}_{2 \mathrm{~h}}$ symmetry) and the corresponding vertical electronic excitation energy of the first excited state $S_{1}$ are summarized in Table II. Thereby, in the NA-QMD-H method the first excited state $S_{1}$ of ethylene is given by the bare single-particle excitation from the highest occupied molecular orbital (HOMO) to the lowest unoccupied molecular orbital (LUMO) of the ground state $S_{0}$, i.e.. in this case a $\pi \rightarrow \pi^{*}$ transition.

The results are compared to those calculated with quantum chemical results (MR-CISD) taken from Ref. [60] as well experimental values (see references in Ref. [27]) We present also additional RHF-CIS results calculated with GAMESS [80] as in the case of the methaniminium cation (see Appendix A). Table II reveals that the corresponding bond lengths $R_{\mathrm{CC}}$ and $R_{\mathrm{CH}}$ and the bond angle $\phi$ are in good agreement with the quantum chemical calculations as well as the experiments. While RHF-CIS overestimates the excitation energy $\Delta E$ of the first excited state $S_{1}$ the respective UHF value of this work is closer to the exact result.

The potential energy curves of the ground and the first two excited states dependent on the torsion angle $\theta$

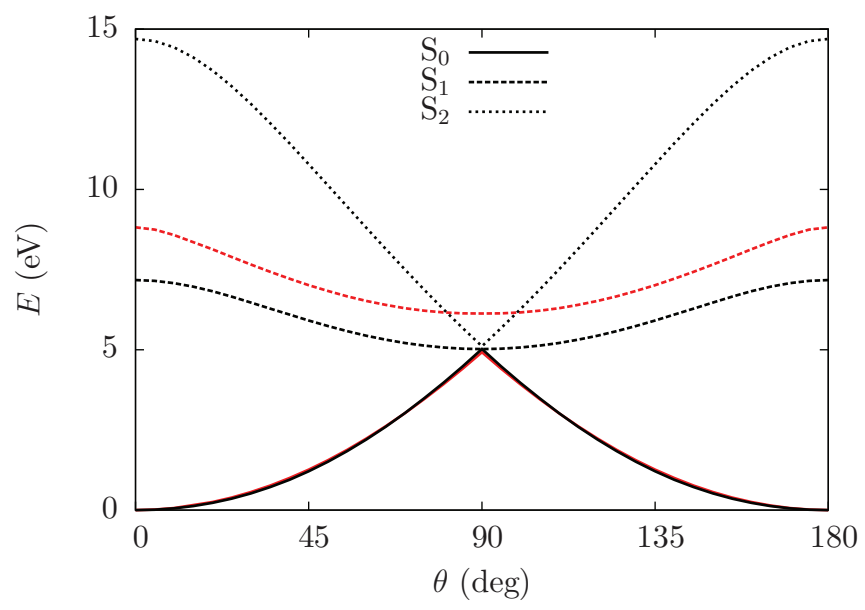

FIG. 8. (Color online) Potential energy surfaces of $\mathrm{C}_{2} \mathrm{H}_{4}$ for the ground state $S_{0}$ and the lowest excited states $S_{1}$ and $S_{2}$ dependent on the torsion angle $\theta$ calculated using UHF (this work) (black curves) and RHF-CIS (GAMESS) (red curves). All remaining geometry parameters are kept frozen according to the ground state equilibrium values. Note that the $S_{2}$ state is missing within RHF-CIS due to its doubly excited character.

(black lines) are displayed in Fig. 8 and compared with the RHF-CIS results calculated with GAMESS [80] (red lines). The first excited state $S_{1}$ exhibits a maximum at the planar ground state equilibrium geometry $(\theta=0)$, whereas the region of the $S_{1} / S_{0}$ conical intersection is indicated by the maximum of the ground state and minimum of the first excited state at $\theta=90^{\circ}$. The RHF-CIS results show a similar trend of the $S_{0}$ and $S_{1}$ surfaces, but exhibiting a energy gap at $\theta=90^{\circ}$ in agreement with more sophisticated quantum chemical MR-CISD calculations [60] for this specific geometry, which is, however, of minor relevance for the general isomerization dynamics we are interested in. The ground state surfaces $S_{0}$ of both methods are nearly indistinguishable due to fact that the spin-unrestricted and spin-restricted Hartree-Fock calculations have the same solution for the shown equilibrium geometries with only varying torsion angle.

Note that in the RHF-CIS results the second excited state $S_{2}$ is missing due to its doubly excited character. This partly contradicts the original concern [81], that bare Kohn-Sham (or Hartree-Fock) excitations (as used in our approach) are generally inferior compared to CIS resp. linear-response TDDFT methods, as doubly excited states might be important for the dynamics as well.
[1] A. R. Olson, Trans. Faraday Soc. 27, 69 (1931).

[2] A. Warshel and M. Karplus, Chem. Phys. Lett. 32, 11 (1975).

[3] J. Michl and V. Bonačić-Koutecký, Electronic Aspects of Organic Photochemistry (Wiley, New York, 1990).

[4] M. Klessinger and J. Michl, Excited States and Photochemistry of Organic Molecules (Wiley- VCH, New York, 1995).

[5] W. Domcke and G. Stock, Adv. Chem. Phys. 100, 1 (1997).

[6] A. H. Zewail, J. Phys. Chem. A 104, 5660 (2000).
[7] F. C. De Schryver, S. De Feyter, and G. Schweitzer (eds.), Femtochemistry (Wiley-VCH, Weinheim, 2001).

[8] D. R. Yarkony, Chem. Rev. 112, 481 (2012).

[9] N. J. Turro, V. Ramamurthy, and J. C. Scaiano, Modern Molecular Photochemistry of Organic Molecules (University Science Books, Sausalito, CA, 2012).

[10] M. Irie, Chem. Rev. 100, 1683 (2000).

[11] A. Warshel, Nature (London) 260, 679 (1976). 
[12] D. Polli, P. Altoe, O. Weingart, K. M. Spillane, C. Manzoni, D. Brida, G. Tomasello, G. Orlandi, P. Kukura, R. A. Mathies et al., Nature (London) 467, 440 (2010).

[13] A. Natansohn and P. Rochon, Chem. Rev. 102, 4139 (2002).

[14] T. Nägele, R. Hoche, W. Zinth, and J. Wachtveitl, Chem. Phys. Lett. 272, 489 (1997).

[15] T. Hugel, N. B. Holland, A. Cattani, L. Moroder, M. Seitz, and H. E. Gaub, Science 296, 1103 (2002).

[16] Y. H. Jiang, A. Rudenko, O. Herrwerth, L. Foucar, M. Kurka, K. U. Kühnel, M. Lezius, M. F. Kling, J. van Tilborg, A. Belkacem et al., Phys. Rev. Lett. 105, 263002 (2010).

[17] Y. Dou and R. E. Allen, J. Chem. Phys. 119, 10658 (2003).

[18] K. M. Tait, J. A. Parkinson, S. P. Bates, W. J. Ebenezer, and A. C. Jones, J. Photochem. Photobiol. A 154, 179 (2003).

[19] M. J. Cook, A.-M. Nygård, Z. Wang, and D. A. Russell, Chem. Commun. 1056 (2002).

[20] D. R. Yarkony, Rev. Mod. Phys. 68, 985 (1996).

[21] B. G. Levine and T. J. Martinez, Ann. Rev. Phys. Chem. 58, 613 (2007).

[22] W. Domcke and D. R. Yarkony, Ann. Rev. Phys. Chem. 63, 325 (2012).

[23] S. Hahn and G. Stock, J. Phys. Chem. 104, 1146 (2000).

[24] C. Cattarius, G. A. Worth, H.-D. Meyer, and L. S. Cederbaum, J. Chem. Phys. 115, 2088 (2001).

[25] G. A. Worth and L. S. Cederbaum, Ann. Rev. Phys. Chem. 55, 127 (2004).

[26] M. Ben-Nun, J. Quenneville, and T. J. Martinez, J. Phys. Chem. A 104, 5161 (2000).

[27] M. Ben-Nun and T. J. Martinez, Chem. Phys. 259, 237 (2000).

[28] J. Quenneville, M. Ben-Nun, and T. J. Martinez, J. Photochem. Photobiol. A 144, 229 (2001).

[29] H. Tao, T. K. Allison, T. W. Wright, A. M. Stooke, C. Khurmi, J. van Tilborg, Y. Liu, R. W. Falcone, A. Belkacem, and T. J. Martinez, J. Chem. Phys. 134, 244306 (2011).

[30] T. K. Allison, H. Tao, W. J. Glover, T. W. Wright, A. M. Stooke, C. Khurmi, J. van Tilborg, Y. Liu, R. W. Falcone, T. J. Martinez et al., J. Chem. Phys. 136, 124317 (2012).

[31] N. L. Doltsinis and D. Marx, Phys. Rev. Lett. 88, 166402 (2002).

[32] C. F. Craig, W. R. Duncan, and O. V. Prezhdo, Phys. Rev. Lett. 95, 163001 (2005).

[33] E. Tapavicza, I. Tavernelli, and U. Rothlisberger, Phys. Rev. Lett. 98, 023001 (2007).

[34] M. Barbatti, G. Granucci, M. Persico, M. Ruckenbauer, M. Vazdar, M. Eckert-Maksic, and H. Lischka, J. Photochem. Photobiol. A 190, 228 (2007).

[35] T. Nelson, S. Fernandez-Alberti, V. Chernyak, A. E. Roitberg, and S. Tretiak, J. Phys. Chem. B 115, 5402 (2011).

[36] M. El-A. Madjet, O. Vendrell, and R. Santra, Phys. Rev. Lett. 107, 263002 (2011).

[37] X. Li, J. C. Tully, H. B. Schlegel, and M. J. Frisch, J. Chem. Phys. 123, 084106 (2005).

[38] F. Wang, C. Y. Yam, L. Hu, and G. Chen, J. Chem. Phys. 135, 044126 (2011).

[39] A. Ojanperä, V. Havu, L. Lehtovaara, and M. Puska, J. Chem. Phys. 136, 144103 (2012).

[40] D. Mendive-Tapia, M. Vacher, M. J. Bearpark, and M. A. Robb, J. Chem. Phys. 139, 044110 (2013).

[41] T. Kunert, F. Grossmann, and R. Schmidt, Phys. Rev. A 72, 023422 (2005).
[42] J. Handt, T. Kunert, and R. Schmidt, Chem. Phys. Lett. 428, 220 (2006)

[43] R. Mitrić, J. Petersen, and V. Bonačić-Koutecký, Phys. Rev. A 79, 053416 (2009).

[44] I. Tavernelli, B. F. E. Curchod, and U. Rothlisberger, Phys. Rev. A 81, 052508 (2010).

[45] Z.-P. Wang, P. M. Dinh, P. G. Reinhard, E. Suraud, and F. S. Zhang, Int. J. Quant. Chem. 111, 480 (2011).

[46] T. Kunert and R. Schmidt, Eur. Phys. J. D 25, 15 (2003).

[47] M. Uhlmann, T. Kunert, and R. Schmidt, Phys. Rev. E 72, 036704 (2005).

[48] M. Uhlmann, T. Kunert, and R. Schmidt, J. Phys. B 39, 2989 (2006)

[49] F. Calvayrac, P.-G. Reinhard, E. Suraud, and C. Ullrich, Phys. Rep. 337, 493 (2000).

[50] M. A. L. Marques, A. Castro, G. F. Bertsch, and A. Rubio, Comput. Phys. Commun. 151, 60 (2003).

[51] T. Laarmann, I. Shchatsinin, A. Stalmashonak, M. Boyle, N. Zhavoronkov, J. Handt, R. Schmidt, C. P. Schulz, and I. V. Hertel, Phys. Rev. Lett. 98, 058302 (2007).

[52] M. Fischer, J. Handt, G. Seifert, and R. Schmidt, Phys. Rev. A 88, 061403 (2013).

[53] M. Fischer, J. Handt, and R. Schmidt, Paper I of this series, Phys. Rev. A 90, 012525 (2014).

[54] M. Fischer, J. Handt, and R. Schmidt, Paper II of this series, Phys. Rev. A 90, 012526 (2014).

[55] M. Barbatti, A. J. A. Aquino, and H. Lischka, Mol. Phys. 104, 1053 (2006).

[56] V. Bonačić-Koutecký, K. Schöffel, and J. Michl, Theor. Chim. Acta 72, 459 (1987).

[57] S. A. Fischer, B. F. Habenicht, A. B. Madrid, W. R. Duncan, and O. V. Prezhdo, J. Chem. Phys. 134, 024102 (2011).

[58] The nuclear density is given by a normalized sum over all trajectories which are represented by localized (Gaussian) density functions $g\left[x-x_{\text {traj }}(t)\right]$ centered at the actual positions $x_{\text {traj }}(t)$ with $x=R_{\mathrm{CN}}, R_{\mathrm{CC}}$, or $\theta$.

[59] M. Ben-Nun and T. J. Martinez, Chem. Phys. Lett. 298, 57 (1998).

[60] M. Barbatti, J. Paier, and H. Lischka, J. Chem. Phys. 121, 11614 (2004).

[61] M. Barbatti, G. Granucci, M. Persico, and H. Lischka, Chem. Phys. Lett. 401, 276 (2005).

[62] M. Barbatti, M. Ruckenbauer, and H. Lischka, J. Chem. Phys. 122, 174307 (2005).

[63] P. G. Szalay, T. Müller, G. Gidofalvi, H. Lischka, and R. Shepard, Chem. Rev. 112, 108 (2012).

[64] U. Saalmann, Ph.D. thesis, TU Dresden, 1997.

[65] T. Kunert, Ph.D. thesis, TU Dresden, 2003.

[66] G. Granucci, M. Persico, and A. Toniolo, J. Chem. Phys. 114, 10608 (2001).

[67] E. Fabiano, T. Keal, and W. Thiel, Chem. Phys. 349, 334 (2008).

[68] K. Kosma, S. A. Trushin, W. Fuss, and W. E. Schmid, J. Phys. Chem. A 112, 7514 (2008).

[69] M. Shapiro and P. Brumer, Quantum Control of Molecular Processes (Wiley-VCH, Berlin, 2011).

[70] M. Wollenhaupt and T. Baumert, Faraday Discuss. 153, 9 (2011).

[71] X. Xie, S. Roither, M. Schöffler, E. Lötstedt, D. Kartashov, L. Zhang, G. G. Paulus, A. Iwasaki, A. Baltuška, K. Yamanouchi et al., Phys. Rev. X 4, 021005 (2014). 
[72] U. Lorenz, Diplomarbeit, TU Dresden, 2007.

[73] M. Fischer, Diplomarbeit, TU Dresden, 2009.

[74] T. Fiedlschuster, master's thesis, TU Dresden, 2014.

[75] M. Richter, P. Marquetand, J. González-Vázquez, I. Sola, and L. González, J. Chem. Theory Comp. 7, 1253 (2011).

[76] J. J. Bajo, J. González-Vázquez, I. R. Sola, J. Santamaria, M. Richter, P. Marquetand, and L. González, J. Phys. Chem. A 116, 2800 (2012).
[77] Extensible Computational Chemistry Environment Basis Set Database, https://bse.pnl.gov/bse/portal.

[78] D. Feller, J. Comput. Chem. 17, 1571 (1996).

[79] K. L. Schuchardt, B. T. Didier, T. Elsethagen, L. Sun, V. Gurumoorthi, J. Chase, J. Li, and T. L. Windus, J. Chem. Inf. Model. 47, 1045 (2007).

[80] General Atomic and Molecular Electronic Structure System, http://www.msg.ameslab.gov/gamess.

[81] N. T. Maitra, J. Chem. Phys. 125, 014110 (2006). 